\title{
DESEMPENHO DO MODELO PGECLIMA_R NA SIMULAÇÃO DE SÉRIES DIÁRIAS SINTÉTICAS DE RADIAÇÃO SOLAR GLOBAL PARA DIFERENTES LOCALIDADES DO ESTADO DO PARANÁ
}

\author{
FRUTEIRA, Rafaela Steimbach - marfagirl@hotmail.com \\ Dep. Biologia Geral - UEPG/Ponta Grossa-PR \\ LEITE, Maysa de Lima - mleite@uepg.br \\ Dep. Biologia Geral - UEPG/Ponta Grossa-PR \\ VIRGENS FILHO, Jorim Sousa - jvirgens@uepg.br \\ Dep. de Matemática e Estatística - UEPG/Ponta Grossa-PR
}

\begin{abstract}
RESUMO. Tendo em vista a grande relevância da radiação solar na análise climática de uma região, o presente estudo objetiva avaliar o desempenho do modelo PGECLIMA_R na geração de séries diárias sintéticas de radiação solar global para diferentes localidades do estado do Paraná. Foram utilizadas séries históricas diárias de radiação solar global das localidades Cascavel, Guarapuava, Guaraqueçaba, Londrina, Palmas, Paranavaí, Ponta Grossa, cedidas pelo Instituto Agronômico do Paraná-IAPAR, compreendendo um período de 30 anos (1980-2009), que serviram para parametrização do modelo, o qual simulou cinco séries de 30 anos para cada localidade. Para testar a eficiência do modelo, os dados gerados foram analisados pelos testes $t$ e $\mathrm{F}$ ao nível de significância de $5 \%$, para comparar as médias mensais das séries simuladas e observadas e, analisar a variabilidade interanual das médias mensais, respectivamente. Paralelamente, utilizaram-se índices para verificar a correlação e a concordância entre as médias mensais simuladas e observadas. Os resultados mostraram que o PGECLIMA_R apresentou um desempenho muito bom na simulação de dados sintéticos de radiação solar global, visto que reproduziu com muito boa acurácia, as médias mensais históricas e suas tendências anuais. Na simulação da variabilidade interanual das médias mensais, o desempenho do PGECLIMA_R foi considerado razoável, uma vez que, o mesmo não conseguiu simular adequadamente esta variabilidade, o que segundo a literatura, se constitui em um dos problemas dos geradores climáticos a ser melhorado.

Palavras chave: Radiação solar, PGECLIMA_R, simulação de dados climáticos.
\end{abstract}

PERFORMANCE OF THE SIMULATION MODEL PGECLIMA_R DAILY SERIES OF SYNTHETIC GLOBAL SOLAR RADIATION TO DIFFERENT LOCATIONS IN THE STATE OF PARANÁ

\section{ABSTRACT}

Given the great importance of solar radiation in climate analysis of a region, this study aims to evaluate the performance of the model PGECLIMA_R in the generation of synthetic series of daily global solar radiation for different localities in the state of Parana. There were used time series of daily global solar radiation for the locations of Cascavel, Guarapuava, Guaraqueçaba, Londrina, Palmas, Paranavaí and Ponta Grossa, provided by the Instituto Agronômico do Paraná-IAPAR, including a 30-year period (1980-2009), which served to improve the parameterization of the model, that simulated five sets of 30 years for each location. To test the efficiency of the model, the data generated were analyzed by $\mathrm{F}$ tests at a significance level of $5 \%$, to compare the monthly averages of the simulated and observed series and analyze the inter annual variability of monthly averages, respectively. In parallel, there were used indices to verify the correlation and agreement between simulated and observed monthly averages. The results showed that the PGECLIMA_R performed very well in the simulation of synthetic data of global solar radiation, as it reproduced with very good accuracy, the average monthly historical and annual trends. In simulating the inter annual variability of monthly averages, the performance of PGECLIMA_R was considered reasonable, since it failed to properly simulate this variability, which according to the literature, constitutes one of the problems of weather generators to be improved.

Keywords: Solar radiation, PGECLIMA_R, simulation of climatic data

\section{Introdução}

O conhecimento da radiação solar e a sua incidência permitem caracterizar o clima de uma região. A radiação solar está diretamente ligada aos fenômenos meteorológicos e aos componentes atmosféricos, tais como vapor d'água e gases, possuindo também influência sobre os principais processos biológicos e fisiológicos na Terra. Também é de grande importância nos estudos agronômicos, na estimativa de mudanças climáticas e na análise dos impactos ambientais nos diferentes ecossistemas. Além disso, a radiação solar pode ser 
utilizada na produção de energia para diferentes aplicações, como energia elétrica, energia para máquinas bioconversoras e aquecedores (VIRGENS FILHO et al., 2005).

Modelos computacionais para simulação de dados climáticos estimam variáveis climáticas, que têm como objetivo gerar um conjunto de valores numéricos, denominado série sintética, com as mesmas características estatísticas da série histórica (EVANGELISTA et al., 2006). As séries sintéticas de dados climáticos geradas são úteis em estudos de mudanças climáticas sobre o potencial e o risco agrícola (SEMENOV \& BARROW, 1997), a perda de água no solo (CECÍLIO et al., 2004), e também na área da ecologia, conforme Kittel et al. (1995), que aplicou um gerador de dados climáticos na construção de um banco de dados bioclimáticos, o qual possibilitou a análise da sensibilidade de um ecossistema às mudanças climáticas.

Vários modelos para geração de séries sintéticas de dados climáticos, que simulam dentre outras variáveis climáticas a radiação solar, foram desenvolvidos. Na literatura sobre o assunto destacam-se o WGEN (RICHARDSON \& WRIGHT, 1984), o WEATHERMAN (PICKERING et al., 1994), o CLIGEN (NICKS et al., 1995) e o LARS-WG (SEMENOV \& BARROW, 1997). Entretanto, muito desses simuladores não possuem bom desempenho para áreas mais abrangentes, como as regiões brasileiras por exemplo, uma vez que seus parâmetros muitas vezes estão calibrados para algumas regiões e suas características. Por este motivo, tornou-se necessário o desenvolvimento de geradores que representassem melhor as condições edafoclimáticas brasileiras. Sendo assim, no Brasil, foram desenvolvidos os modelos GEPAC (VIRGENS FILHO, 1997), SEDAC_R (VIRGENS FILHO, 2001), CLIMABR (BAENA et al., 2005) e PGECLIMA_R (VIRGENS FILHO et al., 2011a; VIRGENS FILHO et al., 2011b).

Nota-se que os geradores de dados climáticos, promovem uma interação de natureza probabilística do clima com componentes de outros sistemas, que fornece uma metodologia para medir o risco de incerteza climática que está relacionada com gerenciamentos alternativos em empreendimentos com agroecossistemas. Portanto, conforme preconiza Zanetti et al. (2006), torna-se necessário que esses dados simulados passem por uma avaliação, a fim de analisar a sua confiabilidade e a representação das condições climáticas reais do local de interesse. Em outras palavras, significa ter a garantia que as propriedades estatísticas contidas nas séries históricas de cada variável climática, foram preservadas.

Tendo em vista a importância dos geradores de dados climáticos nos processos decisórios relacionados ao gerenciamento de agroecossistemas e, o fato de que a radiação solar é uma variável climática determinante no desenvolvimento da vida na Terra, este trabalho objetivou avaliar o desempenho do modelo PGECLIMA_R na simulação de séries diárias de radiação solar global, no que diz respeito à reprodução da média mensal e sua variabilidade interanual, para diferentes localidades do estado do Paraná.

\section{Material e métodos}

Foram utilizadas séries históricas diárias de radiação solar global de 7 localidades paranaenses (Tabela 1) provenientes de estações meteorológicas pertencentes ao Instituto Agronômico do Paraná - IAPAR. Conforme pode ser observado na Figura 1, a seleção das localidades disponíveis tenta abranger na medida do possível, diferentes regiões climato-geográficas para análise do desempenho do modelo PGECLIMA_R no estado do Paraná. 
Tabela 1.

Coordenadas geográficas das localidades paranaenses selecionadas.

\begin{tabular}{lccc}
\hline Localidade & Latitude (S) & Longitude (W) & Altitude (m) \\
\hline Cascavel & $24^{\circ} 26^{\prime} \mathrm{S}$ & $53^{\circ} 26^{\prime} \mathrm{W}$ & $760 \mathrm{~m}$ \\
Guarapuava & $25^{\circ} 21^{\prime} \mathrm{S}$ & $51^{\circ} 30^{\prime} \mathrm{W}$ & $1058 \mathrm{~m}$ \\
Guaraqueçaba & $25^{\circ} 16^{\prime} \mathrm{S}$ & $48^{\circ} 32^{\prime} \mathrm{W}$ & $40 \mathrm{~m}$ \\
Londrina & $23^{\circ} 22^{\prime} \mathrm{S}$ & $51^{\circ} 10^{\prime} \mathrm{W}$ & $585 \mathrm{~m}$ \\
Palmas & $26^{\circ} 29^{\prime} \mathrm{S}$ & $51^{\circ} 59^{\prime} \mathrm{W}$ & $1100 \mathrm{~m}$ \\
Paranavaí & $23^{\circ} 05^{\prime} \mathrm{S}$ & $52^{\circ} 26^{\prime} \mathrm{W}$ & $480 \mathrm{~m}$ \\
Ponta Grossa & $25^{\circ} 13^{\prime} \mathrm{S}$ & $50^{\circ} 01^{\prime} \mathrm{W}$ & $880 \mathrm{~m}$ \\
\hline & & & \\
\hline
\end{tabular}

Figura 1. Localidades paranaenses avaliadas.

As séries sintéticas diárias de radiação solar global, quantificadas em megajoules (MJ), foram geradas pelo modelo PGECLIMA_R a partir dos dados históricos diários. As séries históricas das localidades compreenderam um período de 30 anos (1980-2009), que serviram para parametrização do modelo, o qual simulou outras cinco séries de 30 anos. Para cada localidade foram geradas 60 séries ( 12 meses $x$ cinco simulações). Como foram analisadas sete localidades do Paraná, ao final tem-se um total de 420 séries (sete localidades $x$ 60 séries). Para análise dos dados gerados pelo PGECLIMA_R, os mesmos foram separados em dias chuvosos (com ocorrência de precipitação pluviométrica) e dias secos (sem ocorrência de precipitação pluviométrica). Para testar a eficiência do modelo, os dados gerados foram computados e analisados através dos testes $t$ e teste $\mathrm{F}$, onde o primeiro foi usado para comparar as médias mensais simuladas com as observadas e, o segundo para análise da variabilidade mensal e interanual das médias mensais simuladas e observadas 
para cada localidade. Ambos os testes foram efetuados empregando-se um nível de significância de $5 \%$.

Paralelamente, para verificar a correlação entre as médias mensais simuladas e observadas, determinou-se os valores do coeficiente de correlação de Pearson " $r$ ", do índice de concordância "d" (WILLMOTT et al., 1985) e do índice de desempenho "c" (CAMARGO \& SENTELHAS, 1997).

Segundo Camargo \& Sentelhas (1997), o coeficiente de correlação (Eq.1) indica o grau de dispersão dos dados obtidos em relação à média.

$$
r=\frac{\sum_{i=1}^{n} x_{i} y_{i}-\frac{\sum_{i=1}^{n} x_{i} \sum_{i=1}^{n} y_{i}}{n}}{\sqrt{\left(\sum_{i=1}^{n} x_{i}{ }^{2}-\frac{\left(\sum_{i=1}^{n} x_{i}\right)^{2}}{n}\right)\left(\sum_{i=1}^{n} y_{i}{ }^{2}-\frac{\left.\left(\sum_{i=1}^{n} y_{i}\right)^{2}\right)}{n}\right)}}
$$

A exatidão é medida pelo índice " $d$ " o qual é proveniente do afastamento entre os valores observados e simulados, cujos valores variam de zero (nenhuma concordância) a 1 (concordância perfeita), dado pela seguinte equação (Eq.2):

$$
d=1-\frac{\sum_{i=1}^{n}\left(P_{i}-O_{i}\right)^{2}}{\sum_{i=1}^{n}\left(\left|P_{i}-O\right|+\left|O_{i}-O\right|\right)^{2}}
$$

onde "d" é o índice de concordância; "Pi" é o valor simulado; "Oi" o valor observado e "O" a média dos valores observados.

O índice "c" (Eq.3), obtido pelo produto entre os coeficientes " $r$ " e "d", segue como critério de interpretação do desempenho do modelo, conforme as faixas de valores da Tabela 2.

$$
c=r^{*} d
$$


Tabela 2

\section{Critério de interpretação do desempenho do modelo pelo índice "c" proposto por Camargo \& Sentelhas (1997).}

\begin{tabular}{cc}
\hline Valor de "c" & Desempenho \\
\hline$>0,85$ & Ótimo \\
0,76 a 0,85 & Muito bom \\
0,66 a 0,75 & Bom \\
0,61 a 0,65 & Mediano \\
0,51 a 0,60 & Ruim \\
0,41 a 0,50 & Muito ruim \\
$\leq 0,40$ & Péssimo \\
\hline
\end{tabular}

Como análise complementar, também foi realizada uma comparação visual entre as médias mensais observadas e simuladas. Para tanto, foram elaborados gráficos de tendência e gráficos de dispersão, tanto para dias secos como para dias chuvosos.

\section{Resultados e discussão}

Os resultados dos testes $t$ e $F$ relativos às médias mensais de radiação solar global para dias chuvosos são mostrados na Tabela 3. Para os dados de radiação solar global em dias chuvosos, das 420 séries geradas, $38(9,05 \%)$ foram rejeitadas no teste $t$. A localidade de Guarapuava com 15 (25\%) rejeições de 60 no teste $t$, foi a que apresentou o pior desempenho do modelo. No entanto, a localidade de Palmas obteve ótimo desempenho não apresentando rejeições, o que significa que de maneira geral o PGECLIMA_R, demonstrou muito bom desempenho na reprodução das médias mensais em dias chuvosos, nas localidades paranaenses analisadas.

Para o teste $F$, das 420 séries geradas $116(27,62 \%)$ foram rejeitadas, revelando no geral um desempenho satisfatório na recriação da variabilidade interanual das médias. A localidade de Palmas com $32(53,3 \%)$ de 60 no teste $\mathrm{F}$, foi a que se mostrou com o pior desempenho na reprodução da variabilidade interanual, enquanto que na localidade de Ponta Grossa o resultado foi muito bom, diante do fato de que ocorreu apenas 1 rejeição $(1,7 \%)$. As diferenças estatísticas encontradas no teste $F$ entre os dados simulados e observados já eram esperadas, tendo em vista que, segundo observações de Mavromatis \& Hansen (2001), Soltani \& Hoogenboom (2003) e Virgens Filho et al. (2009), a reprodutibilidade da variabilidade interanual se constitui em um dos problemas dos geradores climáticos a ser resolvido. 
Tabela 3

\section{Quantidade de testes estatísticos rejeitados na avaliação do desempenho do PGCLIMA_R, na simulação da radiação global em dias chuvosos.}

\begin{tabular}{lcc}
\hline Localidades & Teste t & Teste F \\
\hline Cascavel & $12(20,0 \%)$ & $11(18,3 \%)$ \\
Guarapuava & $15(25,0 \%)$ & $12(20,0 \%)$ \\
Guaraqueçaba & $3(5,0 \%)$ & $27(45,0 \%)$ \\
Londrina & $3(5,0 \%)$ & $18(30,0 \%)$ \\
Palmas & $0(0,0 \%)$ & $32(53,3 \%)$ \\
Paranavaí & $2(3,3 \%)$ & $15(25,0 \%)$ \\
Ponta Grossa & $3(5,0 \%)$ & $1(1,7 \%)$ \\
\hline TOTAL & $\mathbf{3 8}(9,05 \%)$ & $\mathbf{1 1 6}(27,62 \%)$ \\
\hline
\end{tabular}

A Figura 2 apresenta as curvas geradas pelas médias mensais observadas e simuladas (nas cinco simulações) para dias chuvosos. Por meio da comparação gráfica nota-se que o programa PGECLIMA_R reproduz de maneira bastante acurada, a tendência das médias de radiação solar global para as diferentes localidades paranaenses analisadas. Podem-se identificar para cada localidade pequenas tendências de subestimativa ou superestimativa em alguns meses, sendo Paranavaí a localidade que apresentou maior subestimativa para radiação em dias chuvosos.

Na Figura 3 estão representados os gráficos de dispersão com os valores dos índices estatísticos " $r$ ", " $d$ " e "c" para a radiação solar global em dias chuvosos nas sete localidades. Os valores encontrados para o índice " $r$ " em todas as localidades foram superiores a 0,98 significando que as médias mensais simuladas possuem alta correlação com as médias mensais observadas. Ao analisar-se o índice de concordância " $d$ " são encontrados valores acima de 0,97 para a grande maioria das localidades, exceto para a localidade de Paranavaí que apresentou o menor valor para o índice " $\mathrm{d}$ " com 0,87 indicando um maior afastamento entre as médias simuladas e as médias observadas. Os valores de "d" obtidos são semelhantes aos registrados por Pereira et al. (2002), que obteve um valor de "d" igual a 0,98 no monitoramento do potencial máximo de radiação solar no município de Ponta Grossa, embora não tenha diferenciado entre dias chuvosos e secos. Os valores obtidos para o índice "c" estão acima de 0,85 para todas as localidades, podendo ser considerado "ótimo" o desempenho do programa PGECLIMA_R, conforme critério proposto por Camargo \& Sentelhas (1997). 


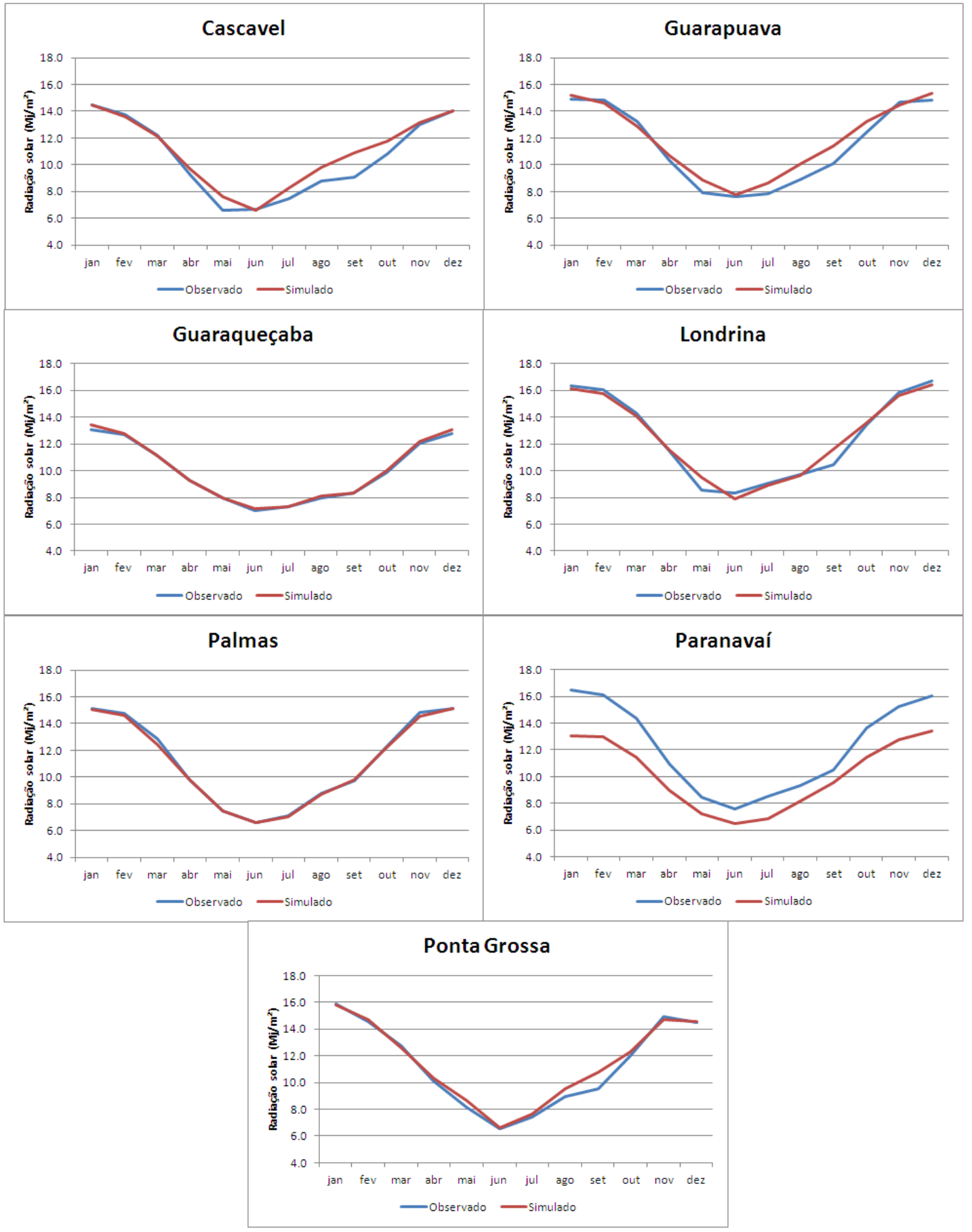

Figura 2

Tendência anual das séries de radiação solar global em dias chuvosos. 


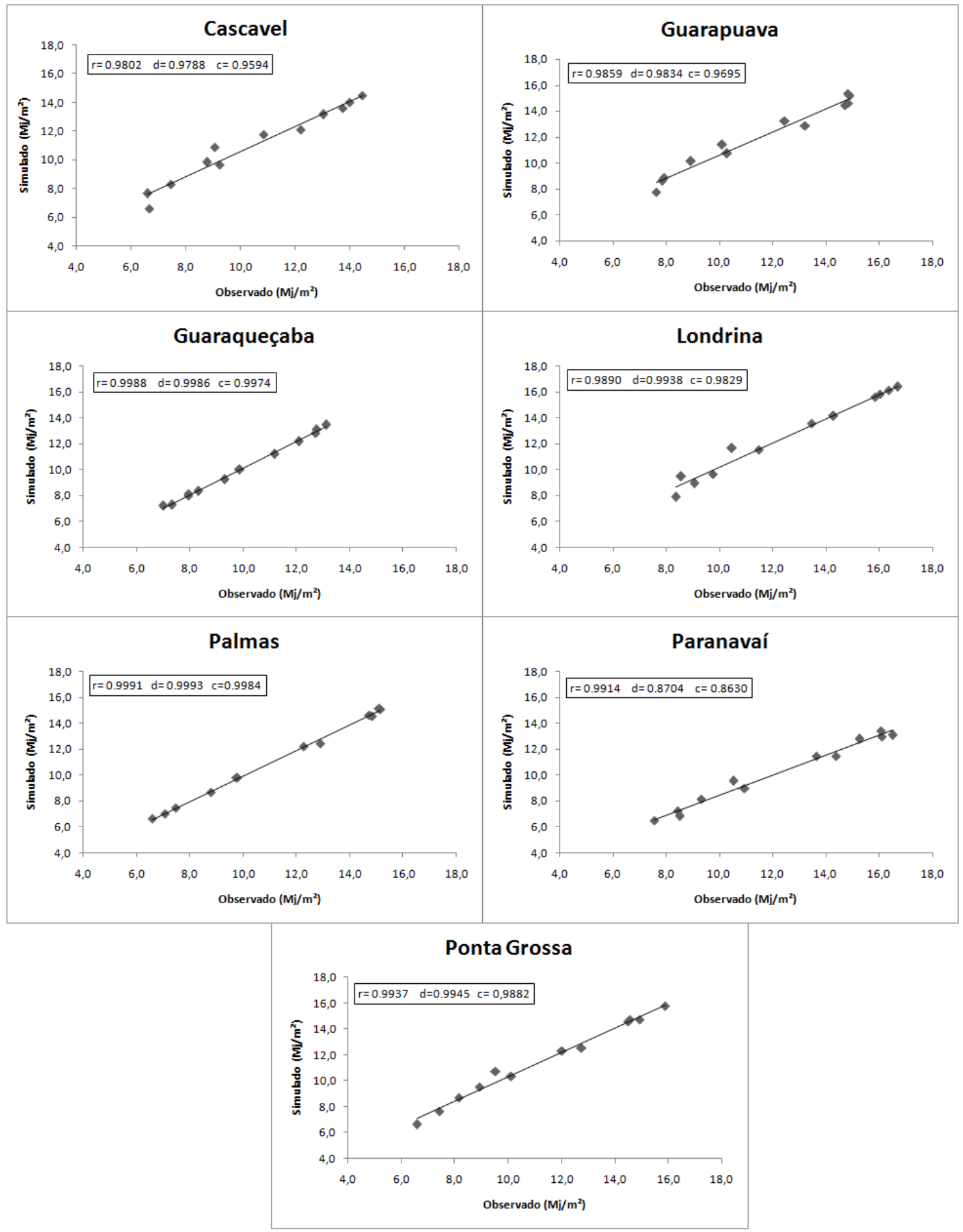

Figura 3

Relação entre as médias mensais simuladas e observadas de radiação solar global em dias chuvosos.

$\mathrm{Na}$ Tabela 4 encontram-se os resultados dos testes $t$ e $\mathrm{F}$ para radiação solar global em dias secos. Das 420 séries geradas para radiação solar global em dias secos, somente $12(2,86 \%)$ foram rejeitados pelo teste $t$. A localidade de Guaraqueçaba, com $9(15 \%)$ rejeições de um total de 60 testes, foi a que apresentou o menor desempenho. Por outro lado, as demais localidades 
apresentaram no máximo 1 rejeição no teste $t$ ao nível de $5 \%$, evidenciando um ótimo desempenho do PGECLIMA_R na reprodução das médias mensais.

Para o teste $F$, das 420 séries geradas 262 (62,38\%) foram rejeitadas, revelando um desempenho ruim na reprodução da variabilidade interanual das médias. A localidade de Palmas apresentou rejeições em todas as simulações realizadas, enquanto que em Ponta Grossa foram encontradas apenas 10 $(16,7 \%)$ de rejeições.

Tabela 4

Quantidade de testes estatísticos rejeitados na avaliação do desempenho do PGCLIMA_R, na simulação da radiação global em dias secos.

\begin{tabular}{lcc}
\hline Localidades & Teste t & Teste F \\
\hline Cascavel & $1(1,7 \%)$ & $13(21,7 \%)$ \\
Guarapuava & $1(1,7 \%)$ & $26(43,3 \%)$ \\
Guaraqueçaba & $9(15,0 \%)$ & $48(80,0 \%)$ \\
Londrina & $0(0,0 \%)$ & $60(100 \%)$ \\
Palmas & $1(1,7 \%)$ & $60(100 \%)$ \\
Paranavaí & $0(0,0 \%)$ & $45(75,0 \%)$ \\
Ponta Grossa & $0(0,0 \%)$ & $10(16,7 \%)$ \\
\hline TOTAL & $\mathbf{1 2}(2,86 \%)$ & $\mathbf{2 6 2}(62,38 \%)$ \\
\hline
\end{tabular}

As curvas geradas pelas médias mensais observadas e simuladas para dias secos encontram-se na Figura 4. Nota-se que a cidade de Paranavaí foi a localidade que obteve maior diferença entre as médias simuladas e observadas apresentado uma tendência de subestimativa generalizada. No entanto, de modo geral, foi possível constatar a eficiência do programa PGECLIMA_R, na reprodução da tendência das médias de radiação solar global em dias secos para as localidades analisadas.

Na Figura 5 estão representados os valores dos índices estatísticos " $r$ ", "d" e "c" para a radiação solar global em dias secos nas sete localidades. Valores superiores a 0,99 para o índice " $r$ ", em todas as localidades, permitem constatar que as médias mensais simuladas possuem alta correlação com as médias mensais observadas. Baena (2004), testando o programa CLIMABR na geração de dados de radiação solar global, obteve para o coeficiente de correlação, para os índices de concordância e de confiança valores acima de 0,98, considerando este um desempenho ótimo.

Quando analisado o coeficiente de concordância " $d$ " referente à exatidão, apenas Paranavaí ("d" $=0,8045$ ) obteve o índice menor que 0,98 , indicando que para esta localidade, houve um maior afastamento dos valores simulados em relação aos observados. Para as demais localidades, os índices mostram que o PGECLIMA_R simulou com acuracidade os dados de radiação solar global para dias secos. Os valores obtidos para o índice "c" estão acima de 0,98 para a maioria das localidades, sendo então considerado desempenho "ótimo", exceto para Paranavaí que em decorrência de um baixo valor do índice "d", apresentou valor "c" de 0,80, que mesmo assim é considerado "muito bom", segundo o critério de interpretação de desempenho proposto por Camargo \& Sentelhas (1997). 


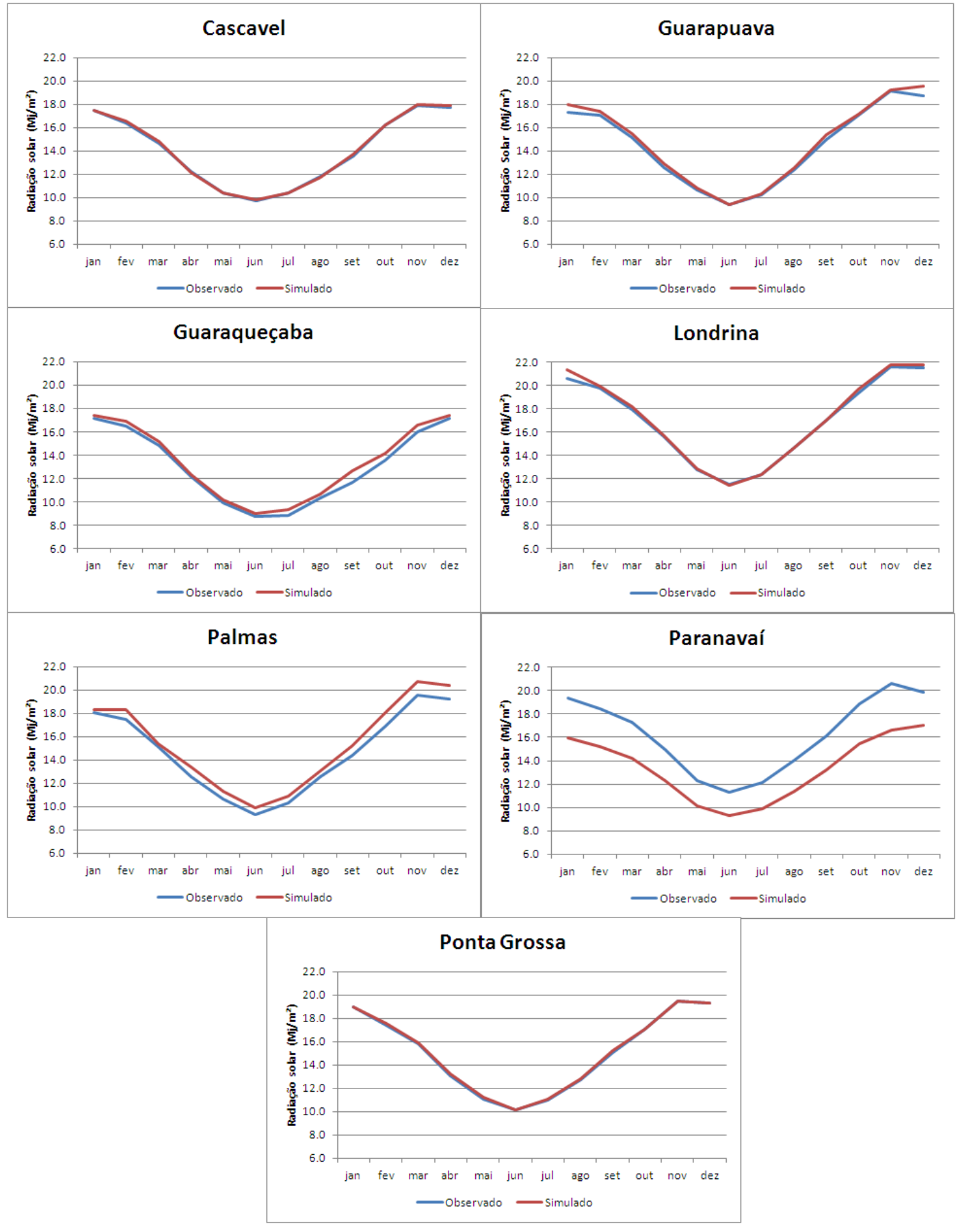

Figura 4

Tendência anual das séries de radiação solar global em dias secos. 


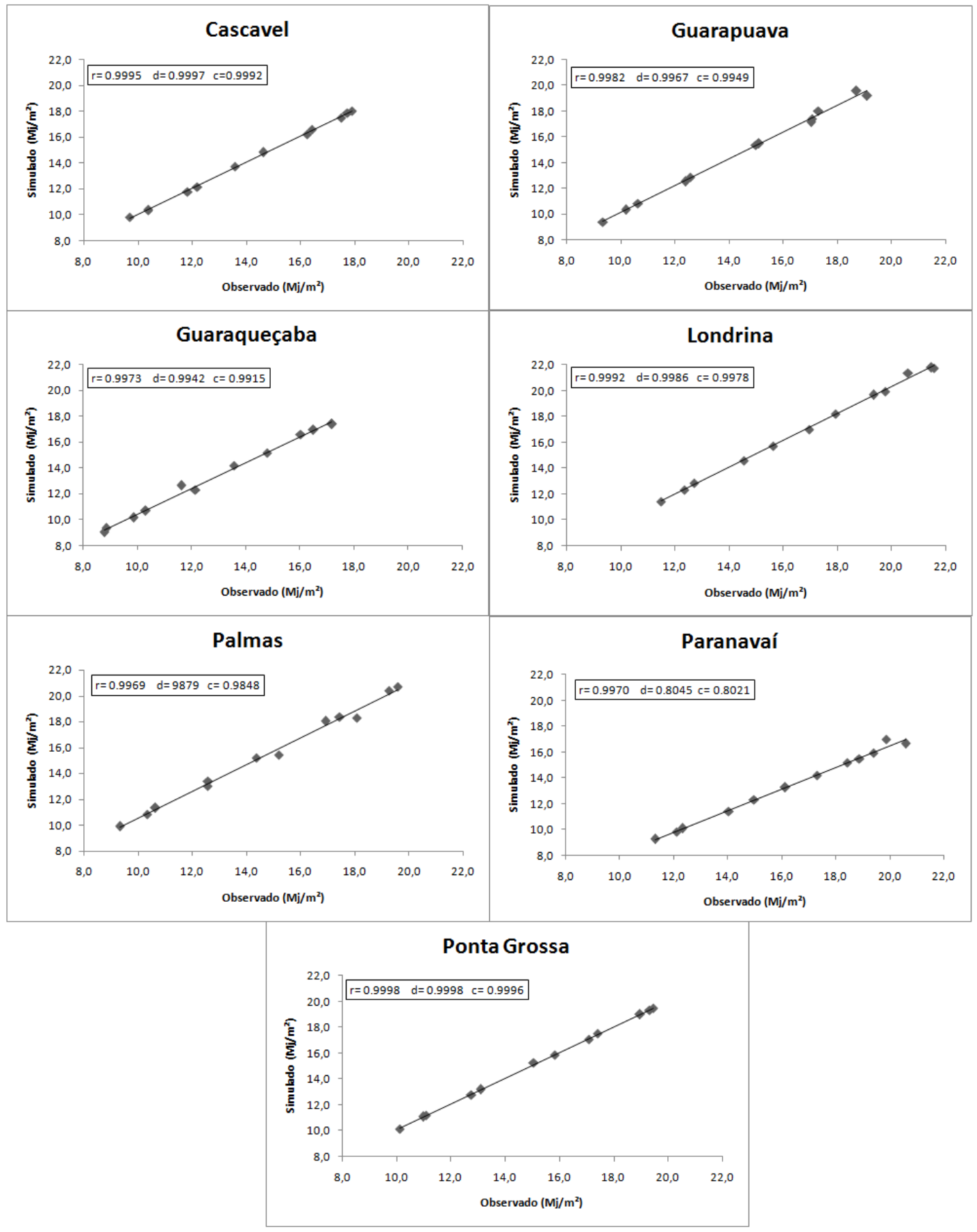

Figura 5

\section{Relação entre as médias mensais simuladas e observadas de radiação solar global em dias secos.}

\section{Conclusões}

Considerando os resultados obtidos, conclui-se que o modelo PGECLIMA_R apresentou um desempenho muito bom na simulação de dados sintéticos de radiação solar global, tanto em dias chuvosos como em dias secos, visto que reproduziu com muito boa acuracidade, as médias mensais históricas e suas 
tendências anuais.

$\mathrm{Na}$ simulação da variabilidade interanual das médias, o desempenho do PGECLIMA_R foi considerado razoável na geração de séries de radiação solar global em dias chuvosos, porém insatisfatório para dias secos, uma vez que, segundo a literatura, a reprodutibilidade da variabilidade interanual se constitui em um dos problemas dos geradores climáticos a ser resolvido.

De maneira geral, o PGECLIMA_R pode ser uma alternativa interessante na geração de dados sintéticos de radiação solar, com grande aplicabilidade para avaliações agronômicas e análise de impactos ambientais. Além disso, também pode ser útil quando utilizado em localidades que não possuem registros históricos de tamanho representativo ou, quando estes apresentarem muitas falhas em suas séries.

\section{Agradecimentos}

Ao CNPq, à Fundação Araucária do Paraná e ao FINEP pelo suporte financeiro, bem como ao IAPAR pela cessão das séries de dados climatológicas.

\section{Referências}

.BAENA, L. G. N. Modelo para geração de séries sintéticas de dados climáticos. Viçosa, 2004. 174 p. Tese (Doutorado em Engenharia Agrícola) Universidade Federal de Viçosa, 2004.

.BAENA, L. G. N; PRUSKI, F. F.; MOREIRA, M. C.; SOUZA, V. B. C.; ZANETTI, S. S., OLIVEIRA, V. P. S. Programa computacional para geração de séries sintéticas de dados climáticos. Engenharia na Agricultura, v.13, n.3, p.210-220, 2005.

.CAMARGO, A. P.; SENTELHAS, P. C. Avaliação do desempenho de diferentes métodos de estimativa da evapotranspiração potencial no Estado de São Paulo, Brasil. Revista Brasileira de Agrometeorologia, Santa Maria, v. 5, n. 1, p.89-97, 1997.

.CECÍliO, R. A.; RODRIGUES, R. D. G.; BAENA. L. G. N.; OliVEIRA, F. G. Analysis of the RUSLE and WEPP models for a small watershed located in Viçosa, Minas Gerais State, Brazil. ISCO - INTERNATIONAL SOIL CONSERVATION ORGANIZATION CONFERENCE, 13., 2004, Brisbane, Austrália. Procedings... Brisbane, 2004. n. 658.

.eVANGELISTA, A. W. P.; FERREIRA, P. A., PRUSKI; F. F. SEDIYAMA, G. C. Avaliação do desempenho do modelo CLIGEN em gerar dados climáticos para modelos hidrológicos e agronômicos. Revista Brasileira de Engenharia Agrícola e Ambiental, Campina Grande, v. 10, n. 1, p.119-127, 2006.

.KITTEL, T. G. F.; ROSENBLOOM, N. A.; PAINTER, T. H.; SCHIMEL, D. S. The VEMAP integrated database for modeling United States ecosystem/vegetation sensitivity to climate change. Journal of Biogeography, Oxford, v.2, p.857862, 1995.

.MAVROMATIS, T.; HANSEN, J. W. Interannual variability characteristics and simulated crop response of four stochastic weather generators. Agricultural and Forest Meteorology, Amsterdan, v.109, p.283-296, 2001.

.NICKS, A. D.; LANE, L. J.; GANDER, G. A. Weather Generator. In: Flanagan, D. C.; Nearing, M. A. USDA-Water erosion prediction project (WEPP). West Lafayette: USDA/ARS, 1995. cap. 2, p.1-22 .

.PEREIRA, A. B.; VRISMAN, A. L.; GALVANI, E. Estimativa da radiação solar global diária em função do potencial de energia solar na superfície do solo. Scientia Agricola, v. 59, n.2, p.211-216, 2002.

.PICKERING, N.B., HANSEN, J.W., JONES, J.W., WELLS, C.M., CHAN, V.K., GODWIN, D.C. WeatherMan: A utility for managing and generating daily 
weather data. Agronomy Journal, v.86, p.332-7, 1994.

.RICHARDSON, C.W., WRIGHT, D.A. WGEN: A model for generating daily weather variables. USDA-ARS ARS-8, 1984, 80p.

.SEMENOV, M. A.; BARROW, E. M. Use of a stochastic weather generator in the development of climate change scenarios. Climatic Change, v.35, p.397-414, 1997.

.SOLTANI, A.; HOOGENBOOM, G. A statistical comparison of the stochastic weather generators WGEN and SIMMETEO. Climate Research, Norwich, v.24, p.215-30, 2003.

.VIRGENS FILHO, J. S. Modelo computacional para simulação de dados climáticos. 1997. 86 p. Dissertação (Mestrado em Agronomia/Energia na Agricultura) - Faculdade de Ciências Agronômicas, Universidade Estadual Paulista, Botucatu, 1997.

.VIRGENS FILHO, J.S. Ferramenta computacional para simulação de séries climáticas diárias, baseada na parametrização dinâmica das distribuições de probabilidade. 2001. 92f. Tese (Doutorado em Energia na Agricultura) - Faculdade de Ciências Agronômicas, Universidade Estadual Paulista, Botucatu, 2001.

.VIRGENS FILHO, J. S.; LEITE, M. L.; CATANEO, A. Simulação de dados diários de radiação solar global utilizando o modelo computacional SIMRAD. In: $V$ Congresso Brasileiro de Agroinformática e II Simpósio Brasileiro de Tecnologia da Informação no Agronegócio Cooperativo, 2005, Londrina, Anais... Londrina, SBI-AGRO, p.883-888.

.VIRGENS FILHO, J. S.; LEITE, M. L.; FRANCO, J. R.; KORELO, M. Modelo computacional estocástico para simulação de séries climáticas diárias de umidade relativa do ar, baseado na parametrização dinâmica das distribuições de probabilidade decorrente da retroalimentação de dados. Revista Brasileira de Climatologia, v.5, p.133-151, 2009.

.VIRGENS FILHO, J. S.; FÉLIX, R. P.; LEITE, M. L.; TSUKAHARA, R. Y. PGECLIMA_R: Gerador estocástico para simulação de cenários climáticos brasileiros. I - Desenvolvimento do gerenciador do banco de dados climáticos. In: XVII Congresso Brasileiro de Agrometeorologia, 2011, Guarapari, Anais... Guaraparí, Sociedade Brasileira de Agrometeorologia, p.1-5, 2011 a.

.VIRGENS FILHO, J. S.; OLIVEIRA, P. M.; LEITE, M. L.; TSUKAHARA, R. Y. PGECLIMA_R: Gerador estocástico para simulação de cenários climáticos brasileiros. II - Automação da análise estatística e validação dos dados simulados. In: XVII Congresso Brasileiro de Agrometeorologia, 2011, Guarapari, Anais... Guaraparí, Sociedade Brasileira de Agrometeorologia, p.1-5, 2011b. .WILLMOTT, C. J.; ACKLESON, S. G.; DAVIS, J. J.; FEDDEMA, K. M.; KLINK, D. R. Statistics for the evaluation and comparison of models. Journal Geography Research, v.90, p, 8995-9005, 1985.

.ZANETTI, S. S.; OLIVEIRA, V. P. S.; PRUSKI, F. F. Validação do modelo ClimaBR em relação ao número de dias chuvosos e à precipitação total diária. Engenharia Agrícola, Jaboticabal, v.26, n.1, p.96-102, 2006. 